\title{
Chemoselectivity in dirhodium(II) catalyzed reactions of diazoacetoacetates prepared from $\alpha, \beta$-unsaturated ketones
}

\author{
Yu Zhang and Michael P. Doyle* \\ Department of Chemistry and Biochemistry, University of Maryland, College Park, Maryland \\ 20742 \\ E-mail: mdoyle3@umd.edu
}

\section{Dedicated to Drs Cynthia A. and Bruce E. Maryanoff in honor of their steadfast dedication to the fields of organic and medicinal chemistry}

\begin{abstract}
$\alpha$-Diazo $\beta$-keto esters from zinc triflate catalyzed Mukaiyama-Michael reactions between $\alpha, \beta$ unsaturated ketones and $\alpha$-silyloxyvinyldiazoacetates are valuable synthons for the synthesis of compounds that can be accessed through catalytic diazo decomposition. Two sets of examples involving $\mathrm{C}-\mathrm{H}$ insertion and aromatic substitution demonstrate the versatility of this methodology.
\end{abstract}

Keywords: Rhodium acetate, C-H insertion, aromatic substitution, Mukaiyama-Michael reaction

\section{Introduction}

We have recently reported that vinyldiazo ester 1 undergoes facile Mukaiyama Michael reactions with $\alpha, \beta$-unsaturated ketones to deliver conjugate addition products in high yield under mild conditions (equation 1). ${ }^{1}$ In this transformation zinc(II) triflate is the preferred catalyst, and a variety of diazoacetoacetates are accessible (Scheme 1). Product yields are high, even with catalyst loading at $0.1 \mathrm{~mol} \%$, and the diazo functionality remains intact. This methodology is superior to other processes that formed the same or similar products by Michael addition. ${ }^{2}$ The ease of this and the related Mukaiyama aldol reaction ${ }^{3,4}$ lies in reaction intermediate 5 (equation 2) that stabilizes the electrophilic addition product to facilitate silyl transfer from the oxygen of the original silyl enol ether to the oxygen of the original unsaturated carbonyl compound, forming the product silyl enol ether 6 (e.g., equation 3) by intramolecular silyl transfer. The silyl enol ether $\mathbf{6}$ undergoes hydrolysis readily in the presence of zinc triflate when open to moisture in the air. We envisioned that the Michael adducts 3 would be valuable synthons for organic synthesis because the mild reaction conditions involved in Mukaiyama- 
Michael reaction should allow installation of an acetoacetate fragment at a late stage of the synthesis. Also, the presence of the remote carbonyl was expected to influence chemoselectivity in diazo decomposition reactions. ${ }^{5}$<smiles>C=C(O[Sb])C(=N)C(=O)OC</smiles>

1<smiles>[X]C(=O)C=C([Z])[Y]</smiles>

2

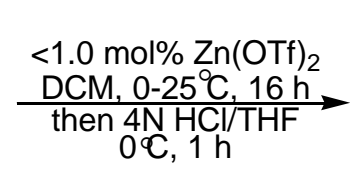

OC, 1<smiles>[X]C(=O)CC([Z])([Y])CC(=O)C(=[W])OC(C)=O</smiles>

3<smiles>COC(=O)C(=[W])CC1CCCC(=O)C1</smiles>

$3 a$<smiles>CC(=O)CC(CC(=O)C(=[W])OC(C)=O)c1ccccc1</smiles>

$3 e$<smiles>CC(=O)C(=[W])C(=O)CC1(C)CCCC(=O)C1</smiles>

$3 b$<smiles>COC(=O)C(=[W])C(=O)CC1CCCC(=O)C1</smiles>

3c

3d<smiles>CC(=O)C(=[W])C(=O)CC(CC(=O)c1ccccc1)c1ccccc1</smiles>

$3 f$<smiles>CCCCCC(CC(C)=O)CC(=O)C(=[W])OC(C)=O</smiles>

$3 g$

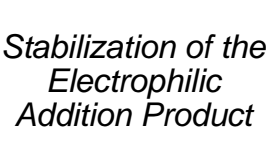
Electrophilic
Addition Product

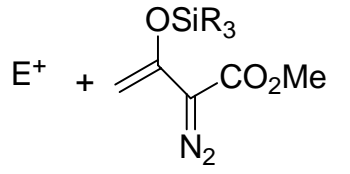

4<smiles>COC(=O)C([NH3+])=C(CF)O[GaH]</smiles>

5

\section{Scheme 1}




\section{Results and Discussion}

Dirhodium carboxylates are well known to effect diazo decomposition of diazoacetoacetates, ${ }^{5}$ and rhodium acetate in catalytic amounts was used to effect diazo decomposition with the intermediate formation of a metal carbene. The diazo-acetoacetates $\mathbf{3 b}$ and $\mathbf{3 g}$ were selected as representative substrates to examine $\mathrm{C}-\mathrm{H}$ insertion reactions and, as expected, they underwent $\mathrm{C}$ $\mathrm{H}$ insertion to give the highly substituted cyclopentane derivatives $\mathbf{7}$ and $\mathbf{8}$ in good yield (equations 4 and 5). These products were obtained following purification by column chromatography. As anticipated from an earlier study of $\mathrm{C}-\mathrm{H}$ insertion reactions of diazoacetoacetates, ${ }^{6}$ the bicyclic compound 7 was isolated as a single diastereoisomer whereas 8 was found to be a mixture of two diastereomers, and the assignment of the relative chemistry was based on the extensive investigations of related substrates by Taber and coworkers. ${ }^{7}$ Rhodium(II) octanoate did not change either the product yield or diastereoselectivity from that obtained with rhodium acetate.. Surprisingly, the more reactive, and sterically more hindered, rhodium triphenylacetate catalyst ${ }^{8}$ did not provide improved yield or selectivity. Consistent with the observation that $\mathrm{C}-\mathrm{H}$ insertion does not generally occur at $\mathrm{C}-\mathrm{H}$ bonds alpha to a carbonyl group, 5,9 no evidence was obtained for such products in dirhodium(II) catalyzed reactions of either $\mathbf{3 b}$ or $\mathbf{3 g}$. Also, insertion into the methyl group of $\mathbf{3 b}$ was not observed.

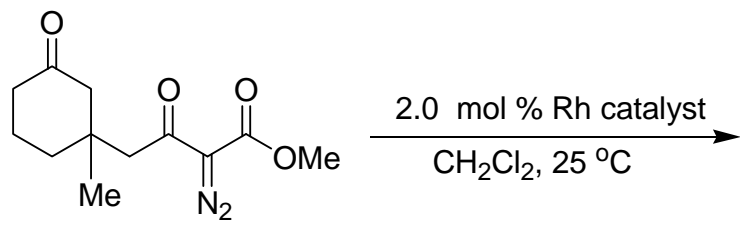

3b

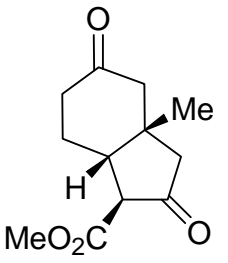

7 (single isomer)

with $\mathrm{Rh}_{2}(\mathrm{OAc})_{4} \quad 61 \%$ yield with $\mathrm{Rh}_{2}(\mathrm{Oct})_{4} \quad 60 \%$ yield

$3 g$

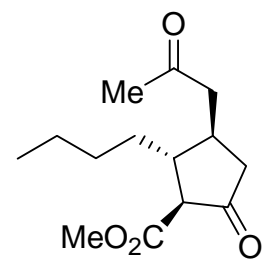

$8 \sim 5: 1 d r(N M R)$

$\begin{array}{ll}\text { with } \mathrm{Rh}_{2}(\mathrm{OAc})_{4} & 61 \% \text { yield } \\ \text { with } \mathrm{Rh}_{2}(\mathrm{Oct})_{4} & 64 \% \text { yield } \\ \text { with } \mathrm{Rh}_{2}(\mathrm{TPA})_{4} & 50 \% \text { yield }\end{array}$

We also found that the diazo-acetoacetates derived from 4-phenyl enones (3e and $\mathbf{3 f}$ ) gave products from aromatic substitution (equations 6 and 7) in reactions catalyzed by dirhodium carboxylates. In contrast to the $\mathrm{C}-\mathrm{H}$ insertion reactions that occur on aliphatic $\mathrm{C}-\mathrm{H}$ bonds, the 
reactions that occur at aromatic $\mathrm{C}-\mathrm{H}$ bonds follow a mechanism of electrophilic addition, followed by hydride transfer from the site of addition to the carbene carbon; ${ }^{10,11}$ and these reactions follow the basic rules associated with electrophilic aromatic substitution. ${ }^{5}$ A low product yield was obtained in the reaction catalyzed by rhodium acetate, but as would be anticipated with the more electrophilic catalysts, dirhodium triphenylacetate ${ }^{8}$ and perfluoroacetate, ${ }^{12}$ aromatic substitution products $\mathbf{9}$ and $\mathbf{1 0}$ were obtained in good yield. Compounds $\mathbf{9}$ and $\mathbf{1 0}$ exist almost entirely in their enol form rather than in their keto form.

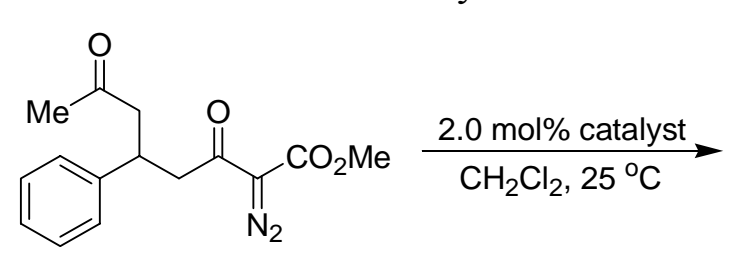<smiles>CC(=O)CC1CC(O)=C(C(C)=O)c2ccccc21</smiles>

$3 e$

$$
\text { with } \mathrm{Rh}_{2}(\mathrm{OAc})_{4}
$$
with $\mathrm{Rh}_{2}(\mathrm{TPA})_{4}$ with $\mathrm{Rh}_{2}(\mathrm{pdb})_{4}$

$$
\begin{aligned}
& 30 \% \text { yield } \\
& 62 \% \text { yield } \\
& 72 \% \text { yield }
\end{aligned}
$$<smiles>CC(=O)C(=N)C(=O)CC(CC(=O)c1ccccc1)c1ccccc1</smiles><smiles>COC(=O)c1ccccc1C(CC(=O)c1ccccc1)CC1=C(O)CCCC1</smiles>

$3 f$

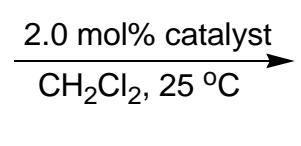

with $\mathrm{Rh}_{2}(\mathrm{TPA})_{4}$ with $\mathrm{Rh}_{2}(\mathrm{pdb})_{4}$
10

$54 \%$ yield $66 \%$ yield

\section{Conclusions}

The use of diazoacetoacetates prepared by Mukaiyama-Michael reactions between $\alpha, \beta-$ unsaturated ketones and the vinyldiazoacetate $\mathbf{1}$ in dirhodium(II)-catalyzed reactions offers a convenient and effective approach to the synthesis of relatively complex multifunctional organic compounds.

\section{Experimental Section}

General. Reactions were performed in oven-dried $\left(140{ }^{\circ} \mathrm{C}\right)$ or flame-dried glassware under an atmosphere of dry $\mathrm{N}_{2}$. Dichloromethane was passed through a solvent column prior to use and was not distilled. Thin layer chromatography (TLC) was carried out using EM Science silica gel $60 \mathrm{~F}_{254}$ plates. The developed chromatogram was analyzed by UV lamp $(254 \mathrm{~nm})$, ethanolate 
phosphomolybdic acid, potassium permanganate $\left(\mathrm{KMnO}_{4}\right)$ or cerium ammonium molybdate (CAM). Liquid chromatography was performed using a forced flow (flash chromatography) of the indicated system on silica gel (230-400 mesh). Rhodium acetate was purchased from Degussa and used as received. Rhodium triphenylacetate ${ }^{13}$ and rhodium perfluorobutyrate ${ }^{8}$ were prepared as described. Rhodium octanoate was used from a sample provided by Professor Al Padwa. Methyl 3-tert-butyldimethylsilanyloxy-2-diazobut-3-enoate was prepared by the method described by Davies. ${ }^{13}$

\section{General procedure for rhodium- catalyzed diazo decomposition}

A solution of the Mukaiyama-Michael adduct $(0.15 \mathrm{mmol})$ in $3.0 \mathrm{~mL}$ of anhydrous $\mathrm{CH}_{2} \mathrm{Cl}_{2}$ was added via syringe pump over $5 \mathrm{~h}$ to a solution of $\mathrm{Rh}_{2}(\mathrm{OAc})_{4}$ or other rhodium catalyst $(0.0030$ mmol) in $3.0 \mathrm{~mL}$ of anhydrous $\mathrm{CH}_{2} \mathrm{Cl}_{2}$ at room temperature. The solvent was removed under reduced pressure, and the catalyst was removed by passing the resulting solution through a short plug of silica. The product was isolated by flash column chromatography (hexanes: ethyl acetate).

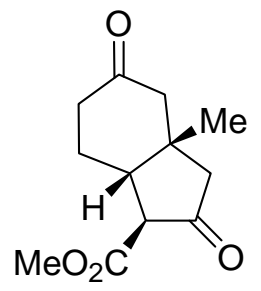

7

1-Methyl-7-carbomethoxybicyclo[4.3.0]nona-3,8-dione 7. Was isolated as a mixture of its enol and keto forms, it relative stereochemistry was assigned based on work by Taber. ${ }^{7} \mathrm{H}$ NMR (400 $\mathrm{MHz}, \mathrm{CDCl}_{3}$ ): $\delta 3.76(\mathrm{~s}, 3 \mathrm{H}), 3.48(\mathrm{~d}, J=12.2 \mathrm{~Hz}, 1 \mathrm{H}), 2.69-2.63$ (m, 1H), 2.42-1.90 (comp, $8 \mathrm{H}), 1.20,(\mathrm{~s}, 3 \mathrm{H})$; (visible enol signal at $10.47 \mathrm{ppm}) ;{ }^{13} \mathrm{C} \mathrm{NMR}\left(400 \mathrm{MHz}, \mathrm{CDCl}_{3}\right): \delta 209.04$, 208.58, 168.89, 56.48, 53.82, 52.79, 49.43, 44.22, 42.18, 35.49, 24.99, 23.48; IR (neat): 1760, 1716, $1667 \mathrm{~cm}^{-1}$; HRMS (FAB) for $\mathrm{C}_{12} \mathrm{H}_{16} \mathrm{O}_{4}[\mathrm{M}+\mathrm{H}]^{+}$calcd: 225.1127; found: 225.1123; TLC R $=0.26(1: 2$ hexanes/EtOAc $)$

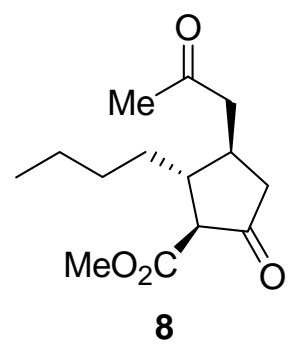

2-Carbomethoxy-3-(n-butyl)-4-(acetylmethyl)cyclopentanone 8. Was isolated as a mixture of diastereomers in $\sim 5: 1$ ratio, and its relative stereochemistry was assigned based on work by Taber. ${ }^{7}$ Compound $\mathbf{8}$ was isolated as a mixture of its enol form and keto form (dominant). Major isomer: ${ }^{1} \mathrm{H}-\mathrm{NMR}\left(400 \mathrm{MHz}, \mathrm{CDCl}_{3}\right): \delta 3.74(\mathrm{~s}, 3 \mathrm{H}), 2.96-2.87(\mathrm{~m}, 1 \mathrm{H}), 2.79-2.71(\mathrm{~m}, 1 \mathrm{H})$, 2.62-2.54 (m, 1H), $2.44(\mathrm{dd}, J=17.6 \mathrm{~Hz}, J=9.4 \mathrm{~Hz}, 1 \mathrm{H}), 2.32-2.22(\mathrm{~m}, 1 \mathrm{H}), 2.17(\mathrm{~s}, 3 \mathrm{H}), 1.92$ 
$(\mathrm{dd}, J=18.6 \mathrm{~Hz}, J=10.6 \mathrm{~Hz}, 1 \mathrm{H}), 1.69-1.61(\mathrm{~m}, 1 \mathrm{H}), 1.39-1.15(\mathrm{comp}, 6 \mathrm{H}), 0.87$ (t, $J=7.2 \mathrm{~Hz}$, $3 \mathrm{H})$; (visible enol signal at $10.43 \mathrm{ppm}) ;{ }^{13} \mathrm{C}$ - NMR (400 $\left.\mathrm{MHz}, \mathrm{CDCl}_{3}\right): \delta 210.08,206.98,170.0$, 61.33, 52.39, 47.40, 45.59, 44.95, 35.22, 32.39, 30.17, 29.03, 22.70, 13.38; IR (neat): 1755, 1720 $\mathrm{cm}^{-1}$; HRMS (FAB) for $\mathrm{C}_{14} \mathrm{H}_{22} \mathrm{O}_{4}[\mathrm{M}+\mathrm{H}]^{+}$calcd: 255.1596; found: 255.1597 ; TLC $\mathrm{R}_{f}=0.23(1: 3$ hexanes/EtOAc). Visible signals for the minor isomer: ${ }^{1} \mathrm{H} \mathrm{NMR}\left(400 \mathrm{MHz}, \mathrm{CDCl}_{3}\right): \delta 2.79-2.70$ $(\mathrm{m}, 1 \mathrm{H}), 2.16(\mathrm{~s}, 3 \mathrm{H}) ;{ }^{13} \mathrm{C} \mathrm{NMR}\left(400 \mathrm{MHz}, \mathrm{CDCl}_{3}\right): \delta 210.87,206.78,169.61,58.96,52.44$, $44.76,43.09,42.44,31.76,30.37,29.49,29.26,22.54$ (one carbon not located).

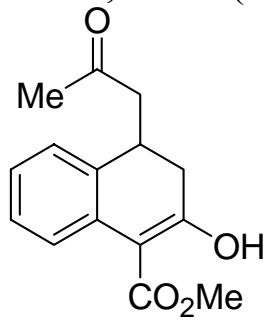

9

1-(Acetylmethyl)-3-hydroxy-4-carbomethoxy-1,2-dihydronaphthalene $\quad 9 .{ }^{1} \mathrm{H} \quad \mathrm{NMR}$ (400 $\mathrm{MHz}, \mathrm{CDCl}_{3}$ ): $\delta 13.27$ (s, 1H), 7.69 (s, $\left.J=7.8 \mathrm{~Hz}, 1 \mathrm{H}\right), 7.24-7.03$ (comp, 3H), 3.91 (s, 3H), 3.43-3.39 (m, 1H), 2.79-2.61 (comp, 3H), 2.39 (dd, $J=16.9 \mathrm{~Hz}, J=3.0 \mathrm{~Hz}, 1 \mathrm{H}) 2.06$ (s, 3H); ${ }^{13} \mathrm{C}$ NMR (400 MHz, $\mathrm{CDCl}_{3}$ ): $\delta$ 206.94, 176.17, 172.18, 135.55, 130.31, 127.15, 126.80, 126.22, 125.43, 99.31, 51.81, 46.49, 34.23, 32.73, 30.71; IR (neat): 2929, 1717, $1636 \mathrm{~cm}^{-1}$; HRMS (FAB) for $\mathrm{C}_{15} \mathrm{H}_{16} \mathrm{O}_{4}[\mathrm{M}+\mathrm{H}]^{+}$calcd: 260.1049; found: 260.1644; TLC $\mathrm{R}_{f}=0.23$ (1:7 hexanes/EtOAc).

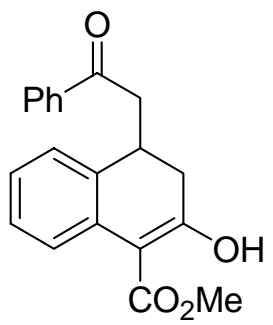

10

1-(Benzoylmethyl)-3-hydroxy-4-carbomethoxy-1,2-dihydronaphthalene $10 .{ }^{1} \mathrm{H}$ NMR (400 $\left.\mathrm{MHz}, \mathrm{CDCl}_{3}\right): \delta 13.29(\mathrm{~s}, 1 \mathrm{H}), 7.88(\mathrm{~d}, J=7.3 \mathrm{~Hz}, 2 \mathrm{H}), 7.72(\mathrm{~d}, J=7.7 \mathrm{~Hz}, 1 \mathrm{H}), 7.53(\mathrm{t}, J=7.4$ $\mathrm{Hz}, 1 \mathrm{H}), 7.41$ (t, $J=7.7 \mathrm{~Hz}, 2 \mathrm{H}), 7.20$ (t, $J=7.3 \mathrm{~Hz}, 2 \mathrm{H}), 7.07$ (t, $J=7.3 \mathrm{~Hz}, 1 \mathrm{H}), 3.93$ (s, 3H), $3.66-3.61(\mathrm{~m}, 1 \mathrm{H}), 3.29(\mathrm{dd}, J=17.4 \mathrm{~Hz}, J=8.4 \mathrm{~Hz}, 1 \mathrm{H}), 3.17(\mathrm{dd}, J=17.4 \mathrm{~Hz}, J=5.5 \mathrm{~Hz}, 1 \mathrm{H})$, $2.85(\mathrm{dd}, J=16.9 \mathrm{~Hz}, J=6.3 \mathrm{~Hz}, 1 \mathrm{H}), 2.55(\mathrm{dd}, J=16.9 \mathrm{~Hz}, J=3.0 \mathrm{~Hz}, 1 \mathrm{H}) ;{ }^{13} \mathrm{C}$ NMR $(400$ $\left.\mathrm{MHz}, \mathrm{CDCl}_{3}\right): \delta 198.31,176.34,172.21,136.82,135.78,133.18,130.46,128.55,128.02$, $127.29,126.83,126.22,125.48,99.37,51.82,41.61,34.25,33.07$; IR (neat): 2927, 1726, 1685 $\mathrm{cm}^{-1}$; HRMS (FAB) for $\mathrm{C}_{20} \mathrm{H}_{18} \mathrm{O}_{4}[\mathrm{M}+\mathrm{H}]^{+}$calcd: 323.1283 ; found: 323.1273 ; TLC $\mathrm{R}_{f}=0.23(1: 7$ hexanes/EtOAc) 


\section{Acknowledgements}

We are grateful to the National Institutes of Health for their support of this research (GM46503).

\section{References}

1. Doyle, M. P.; Liu, Y; Zhang, Y; Jee, N. Org. Lett. 2008, 10,1605.

2. (a) Calter, M. A.; Sugathapala, P, M.; Zhu, C. Tetrahedron Lett. 1997, 38, 3837. (b) Calter, M. A.; Zhu, C. J. Org. Chem. 1999, 64, 1415. (c) Deng, G.; Tian, X.; Qu, Z.; Wang, J. Angew. Chem. Int. Ed. 2002, 41, 2773.

3. Doyle, M. P.; Kundu, K.; Russell, A. E. Org. Lett. 2005, 7, 5171.

4. Kundu, K.; Doyle, M. P. Tetrahedron: Asymmetry 2006, 17, 574.

5. Doyle, M. P.; McKervey, M. A.; Ye, T. Modern Catalytic Methods for Organic Synthesis with Diazo Compounds: From Cyclopropanes to Ylides; John Wiley \& Sons: New York, 1998.

6. Doyle, M. P.; Westrum, L. J.; Wolthuis, W. N. E.; See, M. M.; Boone, W. P.; Bagheri, V.; Pearson, M. M. J. Am. Chem. Soc. 1993, 115, 958.

7. (a) Taber, D. F.; Ruckle, R. E. Jr, J. Am. Chem. Soc. 1986, 108, 7686. (b) Taber, D. F.; You, K. K.; Rheingold, A. L. J. Am. Chem. Soc. 1996, 118, 547.

8. Hashimoto, S.; Watanabe, N.; Ikegami, S. Tetrahedron Lett. 1992, 33, 2709.

9. Doyle, M. P.; Duffy, R.; Ratnikov, M.; Zhou, L. Chem. Rev. 2009, ASAP; DOI: 10.1021/cr900239n.

10. Padwa, A.; Austin, D. J.; Hornbuckle, S. F.; Semones, M. A.; Doyle, M. P.; Protopopova, M. N. J. Am. Chem. Soc. 1993, 114, 1874.

11. Doyle, M. P.; Shanklin, M. S.; Pho, H. Q.; Mahapatro, S. N. J. Org. Chem. 1988, 53, 1017.

12. Doyle, M. P.; Mahapatro, S. N.; Guy, J. K.; Hester, M. R.; VanZyl, C. M.; Boundy, K. L. Inorg. Chem. 1987, 26, 3387.

13. Davies, H. M. L.; Ahmed, G.; Churchill, M. R. J. Am. Chem. Soc. 1996, 118, 10774. 